Castellanos, J.M. (2016). El análisis prosopográfico o la comparación como una vía intermedia para explorar críticamente la diversidad en las élites. Revista de Sociología y Antropología: VIRAJES, 18 (1), 243261. DOI: 10.17151/rasv.2016.18.1.11

\title{
EL ANÁLISIS PROSOPOGRÁFICO O LA COMPARACIÓN COMO UNA VÍA INTERMEDIA PARA EXPLORAR CRÍTICAMENTE LA DIVERSIDAD EN LAS ÉLITES*
}

\section{JUAN MANUEL CASTELLANOS OBREGÓN**}

Recibido: 17 de diciembre de 2015

Aprobado: 27 de febrero de 2016

\section{Artículo de Reflexión}

\footnotetext{
* Reflexión propuesta a partir de la investigación Formas actuales de la movilización armada, en la cual se compararon 68 trayectorias biográficas de agentes inscritos en nueve cuerpos armados activos en el conflicto armado en Colombia en la primera década del siglo XX.

**Antropólogo, con maestría en comunicación educativa y doctorado en ciencias sociales, niñez y juventud. Profesor asociado en el departamento de Antropología y Sociología de la Universidad de Caldas. Juan. castellanos@ucaldas.edu.co. 으 ORCID: 0000-0002-1720-5278
} 


\title{
Resumen
}

Se propone reflexionar acerca de la comparación sistemática de trayectorias vitales de distintos agentes sociales inmersos en espacios de relación social, como una vía para explorar la diversidad de campos de acción social. Esta alternativa resulta especialmente productiva desde la perspectiva teórica estructuralconstructivista que permite mantener la tensión entre la larga y la corta duración de los hechos sociales. Esta aproximación es una especificación sociológica y etnológica de la metodología histórica conocida como la prosopografía o biografía colectiva, aplicada para comprender los procesos de formación de disposiciones y la génesis de grupos, generaciones y ámbitos de acción específicos. Procede mediante la combinación de técnicas de exploración cualitativa que permiten construir un número significativo de casos bien conocidos y la exploración de las cualidades relacionales entre los agentes, sus trayectorias, posiciones y disposiciones en un escenario multifactorial analizado mediante distintas técnicas multivariadas. Con la relación cualitativo-cuantitativo del análisis se trata de dar cuenta de la doble objetividad de la acción social, en tanto estructura estructurada y como práctica con sentido para sus agentes.

Palabras clave: Prosopografía, comparación, análisis de campo, biografía comparada, etnología, élites.

\section{THE PROSOPOGRAPHICAL ANALYSIS OR COMPARISON AS AN INTERMEDIATE WAY TO CRITICALLY EXPLORING DIVERSITY IN ELITES}

\begin{abstract}
It proposes to reflect on the systematic comparison of different social agents vital trajectories immersed in social relationship spaces as a way to explore the diversity of fields. It turns out to be especially productive from the structuralconstructivist perspective. This approach is a sociological and ethnological specification of historical methodology known as prosopography or collective biography applied to understand the formation processes of dispositions and genesis of specific fields of social action. Proceeds by combining qualitative exploration techniques which allows building a significant number of well-known cases and exploring the relational qualities between agents, their trajectories, positions and dispositions in a multifactorial scenario analyzed by multiple relationships analysis and classification analysis. The construction processes clusters and heuristic categories generated by the statistical analysis can be reinstated again in a qualitative framework of understanding the logic of social practices. With this dual qualitative-quantitative relationship is to account for the double objectivity of social action, while structured structure and as a meaning practice to their agents.
\end{abstract}

Key words: Prosopography, comparison, field analysis, comparative biography, ethnology, elites. 


\section{Introducción}

on comunes las referencias a las cualidades compartidas 7 por cohortes generacionales y ocupacionales. La respuesta a referentes estéticos, musicales, políticas y sociales comunes, lo que permite generar condiciones de empatía y comunicación entre personas, que aunque socializadas en contextos geográficos distintos, tienen más cosas en común de las que se podría esperar. Las épocas, los oficios y las profesiones son referentes de subjetivación común, que generan compatibilidades biográficas inesperadas. Sobre eso que es común, en la constitución de semejanzas y diferencias entre contingentes sociales a lo largo de la historia es la preocupación de la entrada metodológica anunciada como la prosopografía. Esta vía analítica tiene como oportunidad de indagación preguntas acerca de quienes hacían parte de algún cuerpo social específico: el clero, los docentes, los oficiales o soldados, una elite o un movimiento en algún concreto de la historia. Parte de la reconstrucción biográfica de personas concretas y su puesta en contraste y comparación con otros semejantes, preguntándose por las condiciones específicas de su movilización, reclutamiento y articulación.

Este interés analítica ha estado centrado en la historia, especialmente en una variante historiográfica centrada en el clero, la nobleza, las clases dominantes y los oficios. A partir de esta tradición, se han incorporado otras miradas disciplinares, especialmente de la sociología y recientemente de la etnografía. En este encuentro de tradiciones es que se haya esta reflexión metodológica, la cual articula también mi propia trayectoria que describiré brevemente, a son de ejemplificar el contexto de producción de esta propuesta.

Mi experiencia investigativa junta varias afinidades, que son en parte las responsables de esta concurrencia metodológica. Mi formación en antropología junta dos estrategias importantes, la etnografía como descripción cualitativa extensa fundada en una experiencia larga y continuada en comunidades, pensadas como unidades pequeñas, aprehensibles en su mayor complejidad, al lado de los procesos de inducción analítica que conducen a la síntesis comprensiva y cuando no a la formalización estructuralista, uno de cuyos ejemplos más visibles es el parentesco. Mi primera producción, mi rito de paso antropológico, fue un ejercicio etnográfico entre los curripaco de la triple frontera entre Colombia, Venezuela y Brasil, en la amazonia noroccidental, alrededor de los procesos de ocupación territorial y la transformación del sistema de parentesco. Este análisis implicó una mirada con distintas escalas espacio-temporales de observación y la composición de una base de datos que incluyó por lo menos 
60 localidades y 840 matrimonios (Castellanos, 1995). Este fue un ejercicio a la vez cualitativo y cuantitativo que implicó la formalización genealógica, la espacialización de las relaciones sociales para pensar la vigencia como la historicidad de las estructuras de parentesco en relación con las estrategias matrimoniales y las formas de ocupación y soberanía territorial.

Luego, al realizar la tesis de maestría propusimos un ejercicio metodológico complementario de análisis del discurso y composición de los procesos de mitificación y ritualización aprehensibles en el contenido y en la forma narrativa, del ejercicio de mediación y de composición de un "orden del mundo", expuesta en las páginas en el periódico de la ciudad en donde resido. La revisión y análisis de periódicos de un año, involucró un ejercicio cualitativo de síntesis y producción de datos y la composición de una base documental de más de 24 mil entradas (Castellanos y Velásquez García, 2003).

El propósito de este manuscrito es mostrar y proponer un ejemplo de investigación complementaria o mixta entre operaciones y procedimientos cualitativos y cuantitativos. Parte de la reflexión de los procedimientos realizados en mi tesis doctoral en la cual analicé, de manera comparativa, la estela de trayectorias de 68 hombres y mujeres jóvenes integrantes de la policía, el ejército, la marina, la vigilancia privada, la guardia carcelaria, las Farc, el Eln y el Epl (2009). Esta reflexión metodológica tiene un carácter básicamente reconstructivo, ninguna alguna aspiración programática, profética o de marketing la orientaron. Desde ese punto de vista espera inscribirse en el debate extenso de la complementariedad, la triangulación y los métodos mixtos a partir del análisis de algunas prácticas investigativas, pensadas como diseños y estrategias con utilidad específica y no como modelos ideales o canónicos de investigación social.

\section{El método}

El método no es fruto de un procedimiento estandarizado. Construye su objetividad en la apertura a la crítica, exponiendo el proceso y los procedimientos a través de los cuales se dice lo que se dice. La oscuridad metodológica sólo es posible en espacios académicos en donde regularmente se hace lo mismo, cuando los procesos están estandarizados o cuando los autores se sienten robustecidos y creen aumentar su consagración ocultando, cual recurso de mago, el ademán que produce la ilusión perceptiva. También hay que echarle la culpa a los editores, que por ampliar la legibilidad eliminan los componentes metodológicos de los textos por oscuros y aburridos. Como en un gesto arquitectónico y estético esconden los cables y las estructuras que soportan los edificios. 
Por lo contrario, presuponemos que si bien en la tarea científica hay mucha creatividad y descubrimiento, su fortaleza está en la capacidad de someter a la crítica el proceso que conduce a ellos. No puede hacerse solamente la evaluación del producto final; acá, como en la escuela, las calificaciones valen. Hay pues que mostrar las cartas, los cálculos, los presupuestos, los encuentros y los desencuentros, así nuestro público sea nuestro más enfermizo competidor. Esas son las condiciones de una crítica comunitaria, para el control social de las condiciones de posibilidad de conocimiento certero, válido, serio y consistente (Bourdieu, 2001, p. 123 y ss).

Podemos considerar la comparación y la complementación como una opción y un tipo particular de diseño metodológico. Una opción metodológica o estrategia específica de construcción y abordaje total del proceso de investigación. No parece una vía acertada separar un momento teórico de un momento metodológico, una metodología para abordar objetos genéricos de conocimiento. Asumimos pues la posición expresada en el "oficio del sociólogo": "Una técnica no es una simple herramienta neutral de recogida de datos, sino teoría en acto: todo artefacto tecnológico supone unos presupuestos teóricos de base" (Bourdieu, Chamboredon y Passeron, 2003 (1973), p. 55). Desde este punto de partida, las perspectivas de comprensión son inseparables de las maneras prácticas de abordar y construir los datum acerca del objeto en cuestión. Luego no haremos referencia a la metodología en la acepción tradicional, como momento práctico de abordaje instrumental de la investigación.

Esta reflexión se desarrollará sobre un tipo de análisis comparativo que tiene relación con varios esfuerzos diferentes. Por un lado el estudio transcultural propuesto por la etnología como método, cuasi-experimental, de comparación controlada y sistemática (Echevarría González, 1990), la cual tiene como uno de sus principales exponentes a G. Murdock (1975, 1972) y uno de sus mayores logros en los archivos de área $\left(\mathrm{HRAF}^{1}\right)$ anidados en la Universidad de Yale. Por otro lado y con este mismo impulso, están los estudios de política comparada desarrollados por la ciencia política de mediados del siglo XX, la cual ha desarrollado una tradición de comparación sistemática con frutos innegables (Almond y Verba, 2001 (1959); Collier, 2003; Inglehart, 1988; Rodríguez-Raga y Seligson, 2007; Sartori, 1992). Así mismo, en el campo de la sociología comparada ha habido importantes avances y propuestas, especialmente desde el desarrollo metodológico planteado por

\footnotetext{
${ }^{1}$ HRAF. Human Relations Area Files o Archivos de estudios de área. Es un programa de investigación desarrollado a partir de la década de 1940, el cual consistió en la construcción de una muestra etnográfica mundial, por áreas culturales, en las cuales se reúnen y sistematizan las principales fuentes etnográficas de las culturas del mundo. http://hraf.yale.edu/
} 
Ragin (2009, 2000) nombrado como small N (Byrne y David Ragin, 2009; Becker y Ragin 1992). Finalmente, en la historia ha habido una tradición de investigación de biografía comparada, nombrada como prosopografía, que es de donde retomamos parte de nuestra inspiración modélica. Pero es del análisis realizado por Bourdieu acerca de la constitución del gusto, del análisis del mercado de la vivienda (2002), el arte y la edición (1988, 1995, 2003) de donde obtenemos la idea principal, que aglutina en una veta teoría y método a través de la idea metodológica del análisis relacional de campo².

La comparación sistemática y controlada de trayectorias vitales de grupos de personas inmersas en campos de acción específicos permite identificar las condiciones históricas de los procesos de reclutamiento, las vías de incorporación, transición y tránsito vital y las reglas propias de constitución de los campos específicos, así como las acciones de producción de subjetividades dispuestas para ello. En la historia de espacios sociales e institucionales específicos, es cuando las cualidades objetivas y subjetivas de los agentes pueden ser comprendidas en su doble articulación: como cosa dada y construida, y como cosa pensada y significada.

El uso de la comparación sistemática es una vía metodológica para dar cuenta tanto de la diversidad como de la homogeneidad, de la condición y la disposición compartida producida por y para ejercicio o acción social situada en contextos histórico-sociales concretos y en espacios institucionales delimitados. Permite, así mismo, explorar la diversidad de las condiciones y disposiciones específicas mediante el control relativo de las combinaciones de cualidades comunes o de la variación de combinaciones de rasgos, variables o categorizaciones. Comparte de alguna manera la idea de que construir cualidades comunes analíticamente, es equivalente en ciencias sociales al ejercicio de experimentación controlada con variables (Sartori, 1992).

\section{La prosopografía y la dinámica de los campos}

El estudio comparado de elites, grupos sociales de una época y región determinada es una metodología histórica y sociológica de uso corriente ${ }^{3}$, que se conoce como el estudio de élites o prosopografía ${ }^{4}$. Se trata de la construcción de biografías colectivas: los banqueros, los dirigentes, los

\footnotetext{
${ }^{2}$ Debo al profesor de la EHSS, Julien Duval haber descubierto en su seminario sobre el "análisis de correspondencias múltiples en sociología" en el 2007, que el análisis bourdieuano era una clase especial de prosopografía.

${ }^{3}$ Ver por ejemplo la compilación de artículos editada por Carasa Soto (Elites. Prosopografia contemporánea 1994).

${ }^{4}$ Prósopon: persona.
} 
concejales, los reclutas, etc., como estrategia de comprensión de una época. Tiene como objetivo entender los procesos de clasificación y producción de clases de edad, gremios, agrupaciones o élites en el espacio y en el tiempo, teniendo en cuenta la división social del trabajo que soporta la existencia de zonas de actividades económicas, políticas, culturales u otras que confieren características comunes a quienes las ocupan. Las estrategias de los individuos aportan una posición en el espacio social dado, adecuado con su manera de ocupar las diferentes posiciones sociales a lo largo del tiempo (Battagliola et al, 1993).

Originalmente fue propuesta como una metodología histórica para la construcción de bancos de datos de época, centrada en la recuperación básica de datos, a partir de personas, teniendo en cuenta su posición genealógica y su posición en el marco de los campos de actividad económica, política, social o religiosa. Combina regularmente dos clases de fichas: las propiedades de la persona, el origen geográfico y social, la generación y la red familiar, la pertenencia confesional, la formación, las cuentas y la profesión para reconstituir la reunión de posiciones ocupadas a lo largo de la vida. La prosopografía puede ser la interpretación de las relaciones internas de un colectivo. Si bien ha tendido a realizarse en investigaciones retrospectivas mediante el análisis de conjuntos de variables uniformes acerca de las vidas de "protagonistas históricos". El método tradicional consistía en "establecer un universo de análisis, formular una serie uniforme de preguntas, combinar la información obtenida, relacionarla, cruzarla y examinarla, buscar variables significativas, situarlas en el contexto de sus correlaciones internas y sus relaciones con otras formas de conducta o de acción" (Carasa Soto, 1994: 46-47).

Esta metodología, desarrollada con alguna predilección por los historiadores franceses, vendría de su voluntad de privilegiar los sujetos como unidades de análisis en sus investigaciones, contrariamente a los investigadores anglosajones, más interesados en las cifras y las valoraciones macro (Beaucarnot, 2004, p. 4). Las fuentes de la prosopografía histórica han sido diversas: archivos, anuarios, actas notariales, en general todas las fuentes que permitan completar los datos biográficos de las personas. Puede ser entendida como una clase de "biografía masiva" (Broady, 2002, p. 58), que se basa en la colecta de un conjunto de información y de propiedades sobre los individuos pertenecientes a un mismo "estamento", en términos weberianos (Weber, 1944 II, p. 686). Su objetivo es la exploración de la estructura y transformación del conjunto, la situación de clase que la engendra.

Para Carasa Soto, en comparación con la historia política y la historia social, la prosopografía reúne una serie de cualidades distintivas. Tiene una 
intención crítica frente al carácter apologético que solía tomar la historia política (Burke, 1996, p. 14). La posición es subjetiva, se pregunta por la media duración frente al interés por el cambio o la permanencia de las otras. Tiene un método que combina la antropología y la sociología, tendría un carácter nomotético, no ideográfico, y deriva de procedimientos deductivos. Su objeto es la relación entre acción y estructura, pregunta por la relación entre agencia humana y agencia social, por las causas particulares y sus correlaciones. Funde sus presupuestos en el análisis de la dominación, logrando resultados de carácter integrador, de tipo mesohistórico, con un tratamiento agregativo de los datos (Carasa Soto, 1994, p. 49). De todas maneras, estas cualidades distintivas de la aproximación de la prosopografía histórica estarán siempre moduladas por la perspectiva teórica que, como contexto de producción y comprensión, las produce. Como en el caso de la clase social, por ejemplo, una cosa será preguntar desde Marx por la emancipación, desde Weber por las variaciones históricas del estatus y la diferenciación social o desde Bourdieu por las oportunidades y los estilos de vida distintivos y diferenciales (Wright et al, 2005, p. 11).

Ese conjunto de acciones analíticas pueden ser incorporadas en un marco de presupuestos teóricos y epistemológicos que traten de superar la dicotomía sociedad-individuo, la oposición macro-micro y, además, se asocien a una teoría de la acción que articule lo estructural u objetivo con lo particular o subjetivo (De Ipola y otros, 2004). Ese marco es el "estructuralismo genético" por un lado y el "estructural constructivismo" por el otro; ambos inspirados en el análisis de campo relacional propuestos por Bourdieu (1991, 1988; Bourdieu y Wacquant, 1995).

La prosopografía propuesta por Bourdieu se basa en el estudio de las cualidades objetivas y subjetivas contrastivas o relacionales de individuos pertenecientes a un mismo campo, recogiendo para ello información como: origen socio-económico, trayectoria socio-profesional, educación, posición en el espacio social, recursos que posee (Beaucarnot, 2004, p. 5). La misma información debe ser colectada para todos los individuos. El objeto de estudio no son solo los individuos, sino la historia y la estructura del campo en el que se encuentran, dando sentido a su trayectoria y a sus destinos sociales.

Como asumimos que la metodología, como diseño específico de investigación, no tiene independencia de la perspectiva y de la problemática en que se formula y delimita el proyecto de investigación, la especificación metodológica de este escrito necesariamente tiene un compromiso teórico particular. Ello no implica que considere que sea imposible una reflexión genérica y, porque no, canónica de procedimientos de investigación, propios de un análisis metodológico. Insisto sí, en que hay mayor productividad del 
pensamiento y la imaginación metodológica si se enseña como estrategia específica y no como modelo general. Como movimientos comunes en coreografías personales.

\section{El procedimiento}

En el ejercicio propuesto para mi tesis se realizaron inicialmente, como exploración cualitativa, 15 entrevistas con jóvenes inscritos en cuerpos armados y 20 con estudiantes de grado 11 que manifestaron su interés de ingresar a la Policía. El análisis de las entrevistas procedió con base en la teoría fundada, con apoyo del programa Atlas.ti (Strauss y Corbin, 2002). Con este proceso de análisis se construyó un marco comprensivo, expuesto en una matriz de operacionalización a partir de la cual se diseñó un cuestionario con alrededor de 560 indicadores. Luego se aplicaron encuestas estructuradas a 68 miembros de 9 cuerpos armados regulares e irregulares que actuaban efectivamente en el conflicto interno colombiano al inicio de este siglo, seleccionados en un proceso de muestreo orientado. El proceso de síntesis de las relaciones estables, de la generación de las categorías y oposiciones entre los conjuntos de cualidades objetivas y subjetivas, y de los agrupamientos o categorías que surgieron, se realizó mediante un análisis multifactorial de análisis de correspondencias múltiples y análisis de clasificación con la ayuda del programa Spad 6, que funciona a partir del "análisis geométrico de datos" propuesto por Benzecri (1973).

Es un análisis que combina, en el caso de la perspectiva teórica estructural constructivista, la génesis, el estado actual y la dinámica propia, especialmente la especificidad del mercado que produce cada subcampo de acción social. El análisis de la génesis de campo implica el presupuesto de la historicidad de los capitales y las posiciones, así como de la estructura del campo, dada por el efecto de histéresis propio de las configuraciones sociales. Así mismo implica un análisis del estado del campo o del contexto en el lenguaje sociológico más genérico. El tercer momento implicaría el análisis específico de las trayectorias de los agentes, de sus cualidades sociales, nombradas como capitales y de sus disposiciones entendidas como esquemas de acción y evaluación y como propensión o ilussio. El cuarto momento implica la constitución de una matriz comparativa, la cual se agrega mediante un proceso de "retroducción", que junta la inducción analítica con la deducción teórica devenida de un marco hipotético específico. Sobre el tercero y cuarto momentos es que se desarrolla especialmente esta reflexión. 
El tipo de análisis prosopográfico se centró en examinar los procesos de "reclutamiento social" 5 de un grupo de personas, quienes al mismo tiempo ocupaban las posiciones más bajas en la jerarquía de diversos cuerpos armados. Por eso propusimos que era una prosopografía al revés, en tanto este grupo no tenía las particularidades de "elite de poder" en que los estudios de este tipo han tendido a centrarse orientados por preguntas por la "clase dominante" desde Marx, o "estatus y clase dirigente" desde Weber o la misma noción de élite desde Pareto, Mosca o Mills (Villa Arranz, 1994, pp. 12-14).

La recolección de la información procedió mediante entrevistas y cuestionario con una muestra variada pero representativa de los agentes eficientes en el campo en cuestión, es decir de las diferentes estructuras que permiten delimitar las principales oposiciones, las posiciones y las especies de capital en juego. Para la construcción del cuestionario se realizaron entrevistas de carácter exploratorio para caracterizar las categorías y el lenguaje para el análisis comparativo entre los sujetos y los campos. Las encuestas conservaron un cuerpo general común asociado a explorar las condiciones sociales y las disposiciones subjetivas de los agentes para la delimitación de categorías cualitativas necesarias para el análisis de campo, requisito del análisis relacional.

La salida analítica de la prosopografía tiene dos vertientes: por una parte, genera un conjunto de relatos y trayectorias vitales que se deben analizar en sí y en relación, de manera cualitativa. La salida cuantitativa del análisis procede a través de correlaciones y correspondencias, para observar cómo se acercan las características o categorías y cómo se atraen estos entre sí los sujetos, conformando clases analíticas, que describen distintas posiciones en el campo objeto de análisis. No se trata solamente de analizar de manera comparativa las condiciones, las disposiciones y los trayectos, sino especialmente, el conjunto de referentes, circunstancias, prácticas y evaluaciones que los sujetos expresan en los relatos de su vida y de las explicaciones de cómo llegaron a ser lo que son y estar en donde están.

\section{Análisis comparativo o de correspondencias - ACM}

Para el análisis comparativo de las encuestas de los “jóvenes guerreros" se desarrolló un ACM (análisis de correspondencias múltiples,

\footnotetext{
${ }^{5}$ Por "reclutamiento social" se entiende la selección de sujetos provenientes de un conjunto limitado de "fracciones de clase" para componer las instituciones, los estamentos y los grupos. No se trata de la acción práctica de reclutamiento por parte de un cuerpo armado. Aunque la primera procede casi siempre por la segunda.
} 
caracterización y tipología de variables, descripción de ejes factoriales, clasificación y jerarquización a través de tablas y árboles de clasificación ${ }^{6}$ ), con miras a establecer la manera cómo se agrupan las distintas categorías, variables y los sujetos. Mediante este tipo de análisis se establece de manera descriptiva la relación entre la variación de las categorías cualitativas y entre los sujetos a partir de la descomposición de la varianza. Este tipo de análisis, introducido por Bourdieu en algunas de sus obras (1988, 1999, 2002), permite identificar las relaciones de fuerza entre las posiciones que ocupan los actores, sus localizaciones en el espacio social y la distribución de las modalidades o cualidades eficientes, teniendo como referente teórico la noción de campo multidimensional y el carácter multiposicionado de las relaciones e interacciones entre los agentes sociales.

El ACM es un tipo de análisis a medio camino entre la descripción y el análisis inferencial, está más cerca de la modelización que del análisis multivariado, aunque algunos manuales de estadística lo incluyan en este capítulo, por ejemplo en Hair et al (1999). Tiene una utilidad heurística y no probabilística. Es un tipo de análisis estadístico que no se centra en "dar cuenta de valores medios", sino que "apunta a dar cuenta de la totalidad de la distribución" (Favereau. 2005, p. 305) y de la variabilidad. Con este tipo de análisis espacial de las categorías se obtiene una representación de la estructura de las relaciones o del campo, similar al mapa del espacio social. Tiene un uso exploratorio, pues permite construir un espacio de relaciones entre las categorías, al dibujar el campo y sus oposiciones, delimitarlo, así como establecer agrupaciones a partir de pequeñas poblaciones. Es un tipo de análisis que conecta las preguntas en relación con la estructura pero también con el dinamismo, con los procesos de transformación, pues aunque recoge información en un momento, puede recuperar su historicidad cuando una muestra implica el antes y el futuro del campo.

Con el ACM, a través de un análisis de caso múltiple (Stake, 2007), se puede poner en evidencia la estructura de oposiciones y equivalencias entre agentes, prácticas, acciones y disposiciones, mediante el análisis de la posición que ocupa y ha ocupado un sujeto o agente particular, relacionarlo con su toma de posición y con sus estrategias de acción. El ACM analiza un conjunto de variables categóricas, para estudiar de "forma conjunta las asociaciones entre las variables" y los sujetos (Bécue Bertaut y Valls i Marsal (s.f.)., p. 46). Parte de la identificación de las propiedades eficientes que pueden ser nombradas como variables pero suelen ser consideradas categorías (expresiones de un sistema de categorización y clasificaciones y no representaciones realistas de algo que varía, como la temperatura o la

\footnotetext{
${ }^{6}$ Una descripción un poco más amplia de estos procesos y sus salidas gráficas en el anexo.
} 
edad). Combina el análisis de categorías cualitativas y cuantitativas, a partir de lo cual se describen y localizan los "agentes eficientes" (Bourdieu 2003, p. 230). El ACM permite la identificación de conjuntos de correlaciones y oposiciones en relación con la posición en el espacio social de los agentes y los capitales que poseen. Busca establecer de manera descriptiva la relación entre las propiedades de los agentes, sus prácticas y concepciones. Este tipo de análisis se realiza a partir de categorías que tienen más de una respuesta nominal o modalidad. Fue desarrollado en Francia a partir de los trabajos de Benzecri (1973), y cuenta con software de respaldo (especialmente el SPAD, aunque hay macros para Excell-Exstad-y SPSS que también permite desarrollar algunos procedimientos).

Este tipo de análisis, clasificado en algunos textos como análisis multivariante, tiene como característica organizar muchos individuos y muchos valores asociados, los cuales son analizados en conjunto (Ganzo (sf), 1). Los análisis multivariados se usan, en general, para procesos de simplificación estructural (reducir la complejidad del problema) mediante la reducción de variables a componentes principales. Así mismo, se le utiliza para la clasificación o agrupación de grupos de individuos homogéneos en relación con grupos de variables similares en su comportamiento. Se complementa con análisis de interdependencia entre grupos de categorías, sin suponer relaciones de causalidad entre ellas (este es el análisis de correspondencias). Los análisis de dependencia explican la relación causal entre grupos de variables, como es el caso de los análisis de regresión (Ganzo (sf), 1-2). El ACM construye un par de matrices adicionales (tabla de frecuencias o contingencia) que establecen las relaciones espaciales entre las varianzas de los datos de cada categoría y entre los sujetos que las poseen.

De este tipo de análisis resultan histogramas ${ }^{7}$ que representan el peso delas categorías en la construcción de los ejes y en la agrupación delos sujetos; gráficas de nube de puntos que distribuyen a los sujetos y a las categorías de acuerdo con su grado de atracción en relación con ejes alineados a centros de inercia móviles entre las varianzas y covarianzas de los datos. Los sujetos quedan así, localizados en un espacio multidimensional equivalente al número de categorías eficientes o en juego, en el cual sus coordenadas son equidistantes a cada una de los valores que estas tienen. Esta es la representación más cercana en el ámbito estadístico a la noción de campo, que es un marco de referencia teórico productivo para comprender los procesos de movilización como engagement (Becker, 2006), como resultado de una íntima relación campo-habitus o espacio social-disposición. Con este tipo se análisis es posible establecer una representación espacial que

\footnotetext{
${ }^{7}$ Una descripción más detallada de los procesos de análisis y sus gráficas en el anexo.
} 
exprese la cercanía y la distancia de los agentes frente a ejes de oposición o interpretación específica. Es la complementación cuantitativa del análisis prosopográfico a partir de la distribución y agrupación de los individuos de acuerdo con la manera como se atraigan las frecuencias de sus cualidades objetivas y de sus respuestas subjetivas. Si bien los sujetos parten clasificados de acuerdo con categorías pre construidas por la perspectiva teórica o por el sentido común, estas son progresivamente reordenadas en categorías de análisis emergentes (o de segundo grado) que componen un paisaje de disposiciones contrastantes. El discurso cualitativo, del relato biográfico y de la experiencia de los sujetos, se complementa así con una representación del espacio de relaciones y oposiciones en que se hallan inmersos.

\section{Entrevistas y cuestionario}

La aplicación práctica de la investigación comparativa se inicia con la construcción de un cuestionario descriptivo del espacio social en estudio, dividido en conjuntos o conglomerados descritos teóricamente. Para el caso de nuestra investigación se propuso la identificación de especies de capital en juego en los diferentes esquemas de acción, percepción y acción. El uso complementario entre el cuestionario, la entrevista y las trayectorias de vida parte de aspiraciones de comprensión que incluyen a la vez conjuntos de datos objetivos o estructurales con conjuntos de datos subjetivos, discursos, representaciones, opiniones, valoraciones o relatos. En los trabajos de Bourdieu se puede tener un ejemplo de este tipo compartido de fuentes de datos múltiples. Asimismo, existe una amplia bibliografía del uso complementario de las dos fuentes y del uso de la entrevista para análisis estructural constructivista (Battagliola y Barthélémy-Proux, 2004; Battagliola et al, 1993; Mauger, 2006).

La aplicación del cuestionario fue uno de los procedimientos de investigación que facilitó un acceso estandarizado de datos y temáticas analizadas para una muestra amplia de sujetos. Las entrevistas abiertas abarcaron en promedio una hora de duración y fueron realizadas en una oficina que contaba con suficientes condiciones de confidencialidad y neutralidad que permitieron su desarrollo sin mayores inconvenientes. Se desarrollaron con una estrategia de entrevista estructurada, con registro sonoro incluido. Solamente la parte final de cuestionario fue diligenciada por los mismos encuestados, aunque no en todos los casos (ejercicio de diferencial semántico sobre el capital agonístico y guerrero). El control de las condiciones de aplicación y la representatividad debe ser adecuado, pues el carácter exploratorio del análisis de correspondencias exige que se 
aplique sobre agentes eficientes y no necesariamente a partir de muestras construidas al azar; es viable a partir de una muestra superior a 40 individuos ${ }^{8}$.

Como el análisis propuesto es descriptivo y correlacional y no causal ni predictivo, el tipo de muestra no tiene que ser representativa de la población. Planteamos la construcción de una muestra teóricamente orientada de casos eficientes de las oposiciones expresadas por las estructuras de movilización que se enfrentan efectivamente en el conflicto interno. En la ausencia de datos internos de composición para todas las fuerzas, era imposible realizar una estimación de una muestra representativa, por lo cual se seleccionó una muestra orientada por condiciones de orden teórico a partir de la identificación de los agentes eficientes ${ }^{9}$.

En un ejercicio de investigación que estamos desarrollando sobre un grupo de "nodrizas" (Chica, 2009), construimos una matriz de la totalidad de la población(90), en la cual sistematizamos la información institucionalmente disponible y la complementamos con un grupo de dimensiones relacionadas con tres categorías básicas de proyecto. Mediante un proceso de análisis de tablas de verdad (Ragin, 2007; Becker, 2009) construimos un conjunto de ocho categorías o clases, de los cuales seleccionamos al azar las mujeres con las cuales hacer el proceso de investigación con enfoque etnográfico (entrevistas, relatos biográficos, observación participante).

La base potencial de la comparación depende de un conocimiento adecuado de los casos, que va más allá de la simple inspección mediante técnicas de survey. Una idea para la comparación y la productividad de los enfoques es propuesta por Ragin (2007), quien propone una mecanismo de contraste entre las perspectivas al cruzar el número de casos o $\mathrm{N}$, con el número de variables o $\mathrm{V}^{10}$.

\begin{tabular}{|c|c|c|}
\hline $\begin{array}{c}\text { Investigación } \\
\text { cualitativa }\end{array}$ & $\mathrm{N}=1$ a 5 & $\begin{array}{c}\mathrm{V}=\alpha . \\
\text { Casi infinita, cuyo caso extremo es la aspiración } \\
\text { holística de la etnografía clásica. }\end{array}$ \\
\hline $\begin{array}{c}\text { Investigación } \\
\text { comparativa }\end{array}$ & $\mathrm{N}=<50>$ & $\begin{array}{c}\mathrm{V}=15 . \\
\text { Un número manejable de variables, que permita } \\
\text { correr modelos multivariados con una potencia } \\
\text { fuerte expresada por el chi cuadrado }\end{array}$ \\
\hline $\begin{array}{c}\text { Investigación } \\
\text { cuantitativa }\end{array}$ & $\mathrm{N}=1500$ & $\begin{array}{c}\mathrm{V}=<5> \\
\text { Un número reducido de casos, que permita sintetizar } \\
\text { el análisis y correr modelos bivariados. }\end{array}$ \\
\hline
\end{tabular}

Traducción a partir de Ragin (2007)

\footnotetext{
${ }^{8}$ Para un ejemplo y discusión al respecto ver Bourdieu (2003).

${ }^{10}$ Esta relación inversa puede ser graficada.
} 
Este carácter intermedio de la investigación comparativa permite mantener el carácter mixto que defendemos y tratamos de describir en este texto. Es especialmente productiva a la hora de explorar la diversidad de las situaciones sociales y para configurar clases analíticas que pueden ser profundizadas cualitativamente. El análisis de clasificación propone principios de oposición y procesos de enclasamiento analítico, cuyo contenido empírico es propuesto por la atracción de las cualidades de los agentes, pero que merece procesos de especificación y profundización. Esta es la idea de complementación o el carácter mixto que proponemos con una vía metodológica intermedia y productiva.

La practica investigativa acostumbrada permite establecer que, por ejemplo en educación, se tienden a hacer muchas etnografías, muchos survey, pero muy poco análisis realmente comparativos con un $n$ suficiente para explorar diversidades (Byrne y David Ragin, 2009). La comparación propuesta acá no es la misma, propuesta por ejemplo por la teoría fundada (Corbin y Strauss, 2002) o por H. Becker (2009), las cuales han sido utilizadas como vía para confirmar teorías cualitativas centradas en el análisis de la homogeneidad de un fenómeno, práctica o experiencia a partir de casos contrastantes.

La clave de la investigación comparativa está en la constitución de una serie suficiente de casos bien conocidos. "Bien conocido" significa en este contexto contar con un detalle cuantitativo y cualitativo adecuado, pero no equivalente a los enfoques típicamente cualitativos como la etnografía, la teoría fundada, la biografía o los estudios de caso (Creswell, 1997). Una imagen de este tipo de condición puede ser atraído de la investigación etnológica, la cual para ser posible necesita de una base etnográfica suficiente e internamente coherente que permita realizar un análisis transcultural (Echevarría González, 1990). Pero no es suficiente una base descriptiva de casos, como se creyó en el proyecto de Murdock de constitución de los HRAF (Murdock, 1975), pues la existencia de descripciones, no implica que permitan construir una matriz comparativa relevante. El empirismo con que se ha tendido a la formulación etnográfica ha sido en parte un obstáculo para la comparación sistemática en antropología y en ciencia política. Es por ello, en parte, que esta vía de investigación ampliamente desarrollada a mediados del siglo XX, fuera lentamente abandonada.

La exploración de las diferencias procede mediante la combinación de técnicas de exploración cualitativa que permiten construir un número significativo de casos bien conocidos y la exploración de las cualidades relacionales entre los agentes (o unidades de análisis específicos: personas, instituciones, países, comunidades), sus trayectorias, posiciones y disposiciones en un escenario multifactorial analizado mediante el análisis 
de correspondencias múltiples y análisis de clasificación. Los procesos de construcción de agrupaciones y categorías heurísticas generados por el análisis estadístico deben ser nuevamente reintegrados en un marco cualitativo de comprensión de la lógica de las prácticas sociales; lo cual se desarrollaría mediante diseños de investigación cualitativa como estudios de caso, biografías, entrevistas a profundidad, observación participante y otros diseños con enfoque etnográfico.

\section{Síntesis de la propuesta}

Una síntesis del diseño metodológico propuesto y desarrollado, como vía analítica que resalta la complementariedad de los estudios cualitativos y cuantitativos, puede ser propuesta en las siguientes etapas:

1. Construcción conceptual del problema. Un uso fuerte de la teoría hasta planteamientos hipotéticos. Aunque no tiene un enfoque monológicodeductivo, y tiene un gran valor en la producción de nueva teoría, esta "nace" en diálogo explícito con las preexistencias.

2. Exploración cualitativa vía enfoque etnográfico (entrevistas semiestructuradas y relatos de trayectoria biográfica). Construcción de una estrategia de muestreo orientado o teórico para dar cuenta de la mayor diversidad y contraste

3. Producción de datos vía survey o entrevistas estructuradas de una base intermedia de casos bien conocidos.

4. Análisis de correspondencias y clasificación a partir de una matriz comparativa.

5. Análisis de clasificación y producción de nuevas categorías, posiciones y disposiciones.

6. Exploración y comprensión cualitativa vía enfoque etnográfico y biográfico de las agrupaciones como unidad de condición y disposiciones sociales que surgieron del proceso de análisis de correspondencias múltiples.

7. Síntesis en un modelo explicativo de los principios de producción de las diferencias en el campo, en las trayectorias y en las disposiciones.

El proceso de complementación entre las cualidades y los procedimientos cualitativos y cuantitativos se produce en la sucesión de las etapas. La triangulación, como efecto de ampliación e incremento de la capacidad de representación, como en una imagen con más pixeles, se produce como proceso de la conversión comprensiva, en tanto cruce 
de interpretaciones posibles surgidas de las exploraciones etnográficas y biográficas y de la comparación, contrastación e identificación de principios de oposición y clasificación de los agentes, sus prácticas y disposiciones mediadas por el análisis de clasificación (lógico o estadístico).

Con esta doble relación entre lo cualitativo y lo cuantitativo se trata de dar cuenta de la doble objetividad de la acción social, en tanto estructura estructurada y como práctica con sentido para sus agentes. Esta doble entrada permite no solo la triangulación, como vía corroborativa, sino la complementación heurística y descriptiva entre las cualidades estructurales y relacionales de las unidades de análisis con la profundidad y ampliación de las particularidades propias de la diversidad social, de las historias inscritas en la historia.

\section{Bibliografía}

Almond, G., y Verba, S. (2001). "La cultura política". En: Diez textos básicos de ciencia política, de VV.AA.. Barcelona: Ariel, (1959).

Battagliola, F., Bertaux-viame, Ferrand I., Michele y Imbert, F. "A propos des biographies: regards croises sur questionnaries et entretiens. En: Populations, no 2 (1993): 325-346.

Battagliola, F. y Barthelemy-proux, T. (2004). «Usages croisés de la généalogie et de la prosopographie: études de cas». En: Bulletin de Liason des sociétes savantes, $\mathrm{n}^{0} 9$.

Beaucarnot., J. (2004). «Généalogie et prosopographie.» En: Bulletin des liason des sociétés savantes 9: 4-5.

Becker, H. «Notes sur le concept d'engagement» (Traduction) (2006). En: Revue Traces,: 177192.

Becker, H. y Ragin, Charles C. (1992). What is a case?: exploring the foundations of social inquiry. Cambridge: University of Cambridge.

Becker, H. (2009).Trucos del oficio. Cómo conducir su investigación en ciencias sociales. Buenos Aires: Siglo XXI editores.

Bécue Bertaut, M. y Valls i Marsal, J. Manual de introducción a los métodos factoriales y clasificación con SPAD. Barcelona: Server d'Estadística, Universitat Autónoma de Barcelona., (s.f.).

Benzecri, J.P. L'analyse des données, tome 2: l'analyse des correspondances. (1973). Paris: Bowman.

Betancur, S. y Castellanos, J. M.. (2003). La puerta giratoria. La deserción escolar en Manizales. En: Cuaderno de Investigación No.1. Manizales: Centro Editorial Universidad de Caldas..

Bourdieu, P. (1991). El sentido práctico. Madrid: Taurus Humanidades. (1988). La distinción: criterios y bases sociales del gusto. Madrid: Taurus.

. (2002). Las estructuras sociales de la economía. $2^{\underline{a}}$ edición. Buenos Aires: Manantial.

. (1995). Las reglas del arte. Génesis y estructura del campo literario. Traducido por Thomas Kauf. Barcelona: Anagrama.

. (2001). Science de la science et réflexivité. Paris: Raison d'agir.

Bourdieu, P. Una revolución conservadora en la edición. (2003). En: Intelectuales, política y poder, de Pierre Bourdieu, 223-264. Buenos Aires: Eudeba,.

Bourdieu, P., Chamboredon, J.C. y Passeron, J. El oficio del sociólogo. (2013). 5a Edición en castellano. Madrid: Siglo veintiuno editores.

Bourdieu, P. y Wacquant, L.. (1995). Respuestas para una antropología reflexiva. Buenos Aires: Grijalbo. 
Broady D. French prosopografy. (2002). En: Pietics 30, no 5-6: 381-385.

Burke, P. Obertura: la nueva historia, su pasado y su futuro. (1996). En: Formas de hacer historia, de Peter (ed.) Burke, 11-37. Madrid: Alianza Universidad.

Byrne, D. y Ragin, Ch. (2009). The SAGE handbook of case-based methods. California: SAGE.

Carasa Soto, P. 4. Recuperación de la historia política y la prosopografía. (1994). En: Elites. Prosopografia contemporánea, 4151. Valladolid: Universidad de Valladolid.

. (1994). Elites. Prosopografia contemporánea. Vallladolid: Universidad de Valladolid, Secretariado de Publicaciones.

Castellanos, J. Bases para una etnografia de la desescolarización. (2005). Memorias de la primera reunión internacional de etnografia y educación: 249-260.

. (2009). Formas actuales de la movilización armada de los y las jóvenes en Colombia. Manizales: Tesis de doctorado en Ciencias Sociales, Niñez y Juventud, Universidad de Manizales-CINDE.

(1995). Parentesco y organización en el espacio. Los Curripaco del río Guainía. Bogotá: Tesis de Antropología, Universidad Nacional de Colombia.

Castellanos, J., Correa, B. y Loaiza, M. Espirales de humo. El acceso de estudios de grupos étnicos en la universidad. (2005). Manizales: Cuaderno de Investigación No. 20, Centro Editorial de la Universidad de Caldas.

Castellanos, J., y Velásquez García B.. La prensa local y el orden del mundo: La Patria en Manizales. (2003). Universidad Tecnológica de Pereira, Pereira: Tesis de Maestría en Comunicación y Educación.

Chica, J. (2009). Un viejo oficio doloroso: nodriza. Proyecto de trabajo de grado en Antropología. Manizales, Universidad de Caldas y FESCO: Bajo la dirección de Juan M Castellanos. Departamento de Antropología y Sociología.

Cisia-Cereste. Systema Spad. Base, Aide a l'interprétation. (2001). Montreuil Cedex France: Centre International de Statistique et d'Informatique Appliquées.

Collier, P et al. (2003). Guerra civil y políticas de desarrollo. Cómo escapar de la trampa del conflicto. Bogotá: Banco Mundial y Alfaomega.

Corbin, J. y Strauss, A. (2002). Bases de la investigación cualitativa: técnicas y procedimientos para la investigación cualitativa. Medellín: Editorial Universidad de Antioquia (http://books. google.com.co/books?id=TmgvTb4tiR8C\&printsec=frontcover\#v=twopage\& $q \& f=f a l s e)$.

Creswell, J. (2007). Qualitative inquiry and research design. Choosing among five traditions. London: SAGE Publications.

De Ipola, E., et al. (2004). El eterno retorno. Acción y sistema en la teoría social contemporánea. Buenos Aires: Editorial Biblos.

Echevarría González, A. (1990). Etnografía y comparación. La investigación intercultural en Antropología. Barcelona: Publications d'Antropologia cultural.

Favereau, O. Complemento: La economía del sociólogo, o pensar (la ortodoxia) a partir de Pierre Bourdieu. (2005). En: El trabajo sociológico de Pierre Bourdieu. Deudas y críticas, de Bernard (dir.) Lahire, 297-368. Argentina: Siglo XXI Editores.

Ganzo, Á. I. «nálisis de datos multivariantes. Introducción al análisis multivariante. (sf). http://dmi.uib.es/ dmiram0/anadadesbio/ADbio0304/apuntesadades.pdf (último acceso: 3 de julio de 2009).

Hair, J.F, et al. (1999). Análisis multivariante. Bogotá, Madrid: Prentice Hall, 5a edición.

Inglehart, R. The Renaissance of Political Culture. (1988). En: American Political Science Review $82, \mathrm{n}^{\circ} 4$.

Mauger, G. (2006). Les bandes, le milieu et la boheme populaire. Paris: Belin.

Murdock, G. (1972). De la structure sociale. Paris: Payot.

Murdock, G. Muestra etnográfica mundial.(1975). En: La Antropología como ciencia, de José LLobera, 203-230. España: Anagrama. 
Ragin, Ch. (2000). Fuzzy-set social science. Chicago: University of Chicago Press.

. (2007). La construcción social de la investigación. Introducción a los métodos y su diversidad. Bogotá: Siglo del Hombre, Universidad de los Andes, SAGE Publications.

Rihoux, B. y Ragin, Ch. (2009). Configurational comparative methods: qualitative comparative analysis (QCA and related techiques). London: SAGE.

Rodríguez-Raga, J. y Seligson, M. Cultura política democráctica en Colombia: 2006. Editado en versión electrónica por: http://sitemason.vanderbilt.edu/lapop/COLOMBIABACK. Vandervillt: Universidad de Vanderbilt, 2007.

Sartori, G. IX. El método de la comparación y la politica comparada. (1992). En: La politica. Lógica y método en las ciencias sociales. Mexico: Fondo de Cultura Ecónómica.

Stake, R. (2007). La investigación con estudios de caso. Madrid: Morata.

Strauss, A. y Corbin, J. (2002). Bases de la investigación cualitativa. Técnicas y procedimientos para desarrollar teoría fundamentada. Medellín: Editorial Universidad de Antioquia, Facultad de Enfermería, Contus.

Villa Arranz, Juan. (1994)1. Clases y elites en la investigación, algunas reflexiones teóricas o metodológicas. En: Elites. Proposografía contemporánea, de Pedro Carasa Soto, 11-34. Valladolid: Universidad de Valladolid.

Weber, M. (1944). Economía y sociedad. 2 vols. México: Fondo de Cultura Económica.

Wright, E. O., Breen, R., Grusky D., Weininger, E., Sorensen, A., y Pakulski, J. (2005). Approaches to Class Analysis . Disponible en: http://www.ssc.wisc.edu/ wright/. Cambridge: Cambridge University Press. 